\title{
Existence and uniqueness for a two-scale system involving tangential operators
}

\author{
M. Amar* - R. Gianni ${ }^{\ddagger}$ \\ * Corresponding author \\ Dipartimento di Scienze di Base e Applicate per l'Ingegneria \\ Sapienza - Università di Roma \\ Via A. Scarpa 16, 00161 Roma, Italy \\ E-mail address: micol.amar@sbai.uniroma1.it \\ $\ddagger$ Dipartimento di Matematica ed Informatica \\ Università di Firenze \\ Via Santa Marta 3, 50139 Firenze, Italy \\ E-mail address: roberto.gianni@unifi.it
}

\begin{abstract}
In this paper we prove existence and uniqueness for a parabolic and a parabolic/elliptic two-scale system of partial differential equations typically arising in homogenization theory or in macroscale-microscale applied models.

KEYWORDS: Abstract parabolic equations, existence and uniqueness, tangential derivatives, homogenization.

AMS-MSC: 35K20, 35K90, 35D30

ACKNowledgement: The first author is member of the Gruppo Nazionale per l'Analisi Matematica, la Probabilità e le loro Applicazioni (GNAMPA) of the Istituto Nazionale di Alta Matematica (INdAM).
\end{abstract}

\section{Introduction}

In this paper we will prove some existence and uniqueness theorems for two different two-scale problems. In this framework, a two-scale problem 
is a system of PDEs involving two unknowns $\left(u, u^{1}\right)$, the first one just depending on a set of space variables (usually called the macroscopic or slow variables) and, possibly, on time $t$, the second one depending also on a second set of spatial variables $y$ (i.e. $u$ depends on $(x, t)$, while $u^{1}$ depends on $(x, y, t))$. This other set of space variables $y$ is usually called the set of microscopic or fast variables.

Such problems have a wide range of applications in many models in which the physical properties at a macroscopic level are affected by phenomena taking place at a microscopic level which, in turn, are affected by the evolution of the macroscopic state variable.

A relevant mathematical framework in which such problems originate is the well-known homogenization theory where, in general, a system of PDEs is studied in a periodic non-homogenous medium, characterized by regions having different physical properties. As the typical length scale of the periodic microstructure tends to zero, we expect to find a "limit" problem satisfied by the macroscopic state variable $u$. Obviously, the microscopic structure of the medium must play a role in the final equations and this is taken into account by the microscopic state variable $u^{1}$, thus leading to the so-called two-scale problem or two-scale system of PDEs (see, among the many others, $[1,2,3,4,5,6,8,9,10,11,12,14]$ and the reference therein). In some recent papers (see $[7,8]$ ), the authors have studied a model for the heat conduction in finely mixed periodic materials made up of two different constituents, having different thermal properties and which are separated by a two-dimensional thermally active interface. Such a problem was treated via homogenization techniques and, as we have described above, it led to a two-scale systems of PDEs of the type studied in this paper. Its main feature is that the equations for the microscopic function $u^{1}$ in the two physically different regions $E_{\text {int }}$ and $E_{\text {out }}$ are coupled via a LaplaceBeltrami equation satisfied on the surface $\mathcal{G}$ separating such two regions. Due to this peculiar structure, the problem for the pair $\left(u, u^{1}\right)$ (see $(3.1)-$ (3.7) and (4.1)-(4.6) below) must be dealt carefully.

In particular, the homogenized limit problem obtained in $[7,8]$ belongs to the family of elliptic/parabolic systems (4.1)-(4.6), whose well-posedness is proved in Section 4 via a relaxation technique. This is done by introducing a new problem in which $u^{1}$ satisfies a parabolic equation in $E_{\text {int }} \cup E_{\text {out }}$ (see (4.9)-(4.15)) and passing to the limit as $\varepsilon$ tends to zero ( $\varepsilon$ being the coefficient of $u_{t}^{1}$ ). In order to achieve our goal, we make use of some a-priori estimates independent of $\varepsilon$ (see (4.19)). 
In turn, the well-posedness of the relaxed system (3.1)-(3.7) is obtained in Section 3 using abstract parabolic theory (see, for instance, [13] and [15]). When problems of type (3.1)-(3.7) or (4.1)-(4.6) appear as homogenization limit, this automatically yields the existence of solutions (leaving only uniqueness to be established). Here, we prove an independent result of existence and uniqueness under more general assumptions; for instance, both in (3.1) and in (4.1), we allowed the matrix $A$ in the principal part to be also negative definite. We believe that these problems are of mathematical interest in themselves and, for this reason, we have decided to devote this paper to their study.

The paper is organized as follows. In Section 2, we recall the definition and some properties of the tangential operators (gradient, divergence, LaplaceBeltrami operator) and we state our geometrical setting. In Sections 3 and 4 , we state and prove our main results.

\section{Prelimineries}

\subsection{Tangential derivatives}

Let $\phi$ be a $\mathcal{C}^{2}$-function, $\boldsymbol{\Phi}$ be a $\mathcal{C}^{2}$-vector function and $S$ a smooth surface in $\mathbb{R}^{N}$ with normal unit vector $n$. We recall that the tangential gradient of $\phi$ on $S$ is given by

$$
\nabla^{B} \phi=\nabla \phi-(n \cdot \nabla \phi) n
$$

and the tangential divergence of $\boldsymbol{\Phi}$ on $S$ is given by

$$
\begin{array}{r}
\operatorname{div}^{B} \boldsymbol{\Phi}=\operatorname{div}^{B}(\boldsymbol{\Phi}-(n \cdot \boldsymbol{\Phi}) n)=\operatorname{div}(\boldsymbol{\Phi}-(n \cdot \boldsymbol{\Phi}) n) \\
=\operatorname{div} \boldsymbol{\Phi}-\left(n \cdot \nabla \boldsymbol{\Phi}_{i}\right) n_{i}-(\operatorname{div} n)(n \cdot \boldsymbol{\Phi}),
\end{array}
$$

where, taking into account the smoothness of $S$, the normal vector $n$ can be naturally defined in a small neighborhood of $S$ as $\frac{\nabla d}{|\nabla d|}$, where $d$ is the signed distance from $S$. Moreover, we define as usual the Laplace-Beltrami operator as

$$
\Delta^{B} \phi=\operatorname{div}^{B}\left(\nabla^{B} \phi\right)
$$

Finally, we recall that on a regular surface $S$ with no boundary (i.e. when $\partial S=\emptyset$ ) we have

$$
\int_{S} \operatorname{div}^{B} \boldsymbol{\Phi} \mathrm{d} \sigma=0
$$




\subsection{Geometrical setting}

Let $\Omega$ be an open connected bounded subset of $\mathbb{R}^{N}$ with Lipschitz boundary. Let us denote by $Y$ the open subset $(0,1)^{N} \subset \mathbb{R}^{N}$ and assume that $Y=$ $E_{\text {out }} \cup E_{\text {int }} \cup \mathcal{G}$, where $E_{\text {int }}$ and $E_{\text {out }}$ are two disjoint open subsets of $Y$, $E_{\text {out }}$ is connected, $\mathcal{G}=\partial E_{\text {int }}=\partial E_{\text {out }} \cap Y, \mathcal{G} \cap \partial Y=\emptyset$ and $\mathcal{G}$ is assumed to be of class $\mathcal{C}^{\infty}$. Finally, let $\nu$ denote the normal unit vector to $\mathcal{G}$ pointing into $E_{\text {out }}$.

We will also use the following notation. Let $T>0$ be a given time, for any spatial domain $G$, we will denote by $G_{T}=G \times(0, T)$ the corresponding space-time cylindrical domain over the time interval $(0, T)$.

\section{Well-posedness for the parabolic two-scale sys- tem}

We consider the following two-scale parabolic problem for the pair $\left(u, u^{1}\right)$ defined by

$$
\begin{aligned}
& u_{t}-\operatorname{div}\left(A \nabla u+\int_{Y} B\left(\nabla_{y} u^{1}+\nabla_{x} u\right) \mathrm{d} y+\int_{\mathcal{G}} \ell\left(\nabla_{y}^{B} u^{1}+\nabla_{x}^{B} u\right) \mathrm{d} \sigma\right)=f, \text { in } \Omega_{T} ; \\
& u_{t}^{1}-\operatorname{div}_{y}\left(B\left(\nabla_{y} u^{1}+\nabla_{x} u\right)\right)=g, \quad \text { in } \Omega_{T} \times\left(E_{\text {int }} \cup E_{\text {out }}\right) \text {; } \\
& \operatorname{div}_{y}^{B}\left(\ell\left(\nabla_{y}^{B} u^{1}+\nabla_{x}^{B} u\right)\right)=-\left[B\left(\nabla_{y} u^{1}+\nabla_{x} u\right) \cdot \nu\right]-h, \quad \text { in } \Omega_{T} \times \mathcal{G} ; \\
& u^{1}(x, \cdot, t) \quad \text { is } Y \text {-periodic for a.e. }(x, t) \in \Omega_{T} \text {; } \\
& u=0 \text {, } \\
& \text { on } \partial \Omega \times(0, T) \text {; } \\
& u^{1}(x, y, 0)=\bar{u}_{0}^{1}(x) \\
& \text { in } \Omega \times Y \text {; } \\
& u(x, 0)=\bar{u}_{0}(x) \text {, }
\end{aligned}
$$

where the notation $[\cdot]$ denotes the jump across the interface $\mathcal{G}$ of the function inside the square brackets.

We assume that $f, g, h, \bar{u}_{0}, \bar{u}_{0}^{1}$ satisfies

$$
\begin{gathered}
f \in L^{2}\left(\Omega_{T}\right), \quad g \in L^{2}\left(\Omega_{T} \times Y\right), \quad h \in L^{2}\left(\Omega_{T} \times \mathcal{G}\right), \\
\bar{u}_{0} \in L^{2}(\Omega), \quad \bar{u}_{0}^{1} \in L^{2}(\Omega \times Y),
\end{gathered}
$$


and that

$$
\begin{aligned}
& A \in\left(L^{\infty}(\Omega)\right)^{N}, A(x) \xi \cdot \xi \geq-\gamma_{A}|\xi|^{2}, \text { for a.e. } x \in \Omega, \forall \xi \in \mathbb{R}^{N}, \\
& B \in\left(L^{\infty}(\Omega \times Y)\right)^{N}, B(x, y) \xi \cdot \xi \geq \gamma_{B}|\xi|^{2} \text {, for a.e. }(x, y) \in \Omega \times Y, \forall \xi \in \mathbb{R}^{N}, \\
& \ell \in L^{\infty}(\Omega \times \mathcal{G}), \ell(x, y) \geq \gamma_{\ell}, \text { for a.e. }(x, y) \in \Omega \times \mathcal{G}
\end{aligned}
$$

where $\gamma_{B}>\gamma_{A}>0$ and $\gamma_{\ell}$ are strictly positive constants.

Remark 3.1. Notice that, as a consequence of (3.2) and (3.3), it follows

$$
\begin{gathered}
\frac{\mathrm{d}}{\mathrm{d} t}\left(\int_{Y} u^{1}(x, y, t) \mathrm{d} y\right)=\int_{Y} g \mathrm{~d} y+\int_{Y} \operatorname{div}_{y}\left(B\left(\nabla_{y} u^{1}+\nabla_{x} u\right)\right) \mathrm{d} y \\
=\int_{Y} g \mathrm{~d} y-\int_{\mathcal{G}}\left[B\left(\nabla_{y} u^{1}+\nabla_{x} u\right) \cdot \nu\right] \mathrm{d} \sigma \\
=\int_{Y} g \mathrm{~d} y+\int_{\mathcal{G}} \operatorname{div}_{y}^{B}\left(\ell\left(\nabla_{y} u^{1}+\nabla_{x} u\right)\right) \mathrm{d} \sigma+\int_{\mathcal{G}} h \mathrm{~d} \sigma=\int_{Y} g \mathrm{~d} y+\int_{\mathcal{G}} h \mathrm{~d} \sigma .
\end{gathered}
$$

Hence, the mean average of $u^{1}$ over the unit cell $Y$ is prescribed in terms of the sources $g$ and $h$ and the initial datum $\bar{u}_{0}^{1}$, i.e.

$$
\int_{Y} u^{1}(x, y, t) \mathrm{d} y=\int_{Y} \bar{u}_{0}^{1}(x, y) \mathrm{d} y+\int_{0}^{t}\left(\int_{Y} g \mathrm{~d} y+\int_{\mathcal{G}} h \mathrm{~d} \sigma\right) \mathrm{d} \tau .
$$

Therefore, replacing $u^{1}$ with the function $\widehat{u}^{1}$ defined by

$$
\begin{aligned}
\widehat{u}^{1}(x, y, t)=u^{1}(x, y, t) & -\int_{Y} \bar{u}_{0}^{1}(x, y) \mathrm{d} y \\
& -\int_{0}^{t}\left(\int_{Y} g(x, y, \tau) \mathrm{d} y+\int_{\mathcal{G}} h(x, y, \tau) \mathrm{d} \sigma\right) \mathrm{d} \tau,
\end{aligned}
$$

we get that the pair $\left(u, \widehat{u}^{1}\right)$ satisfies the problem (3.1)-(3.7) with a new initial datum $\overline{\widehat{u}}_{0}^{1}$ (having null mean average on $Y$ ) defined by

$$
\overline{\widehat{u}}_{0}^{1}(x, y)=\bar{u}_{0}^{1}(x, y)-\int_{Y} \bar{u}_{0}^{1}(x, y) \mathrm{d} y,
$$


and a new source $g$ defined by

$$
\widehat{g}(x, y, t)=g(x, y, t)-\int_{Y} g(x, y, t) \mathrm{d} y-\int_{\mathcal{G}} h(x, y, t) \mathrm{d} \sigma .
$$

Moreover, the sources $\widehat{g}$ and $h$ satisfy the following compatibility condition

$$
\int_{Y} \widehat{g}(x, t, y) \mathrm{d} y+\int_{\mathcal{G}} h(x, t, y) \mathrm{d} \sigma=0, \quad \text { for a.e. }(x, t) \in \Omega_{T}
$$

As a consequence of Remark 3.1, without loss of generality, we will assume that the initial datum $\bar{u}_{0}^{1}$ in (3.6) satisfies the further condition

$$
\int_{Y} \bar{u}_{0}^{1}(x, y) \mathrm{d} y=0, \quad \text { for a.e. } x \in \Omega,
$$

and that the sources $g$ and $h$ satisfies the compatibility condition (3.13), so that, in the pair $\left(u, u^{1}\right)$ of solutions, the function $u^{1}$ satisfies

$$
\int_{Y} u^{1}(x, y, t) \mathrm{d} y=0, \quad \text { for a.e. }(x, t) \in \Omega_{T} .
$$

Assumptions (3.13)-(3.15) will be instrumental in considering problem (3.1)(3.7) in an abstract parabolic setting, as for instance in [13] and [15]. To this purpose, we are led to introduce the following function space

$$
\begin{array}{r}
H_{0, \#}^{1, B}(\Omega \times Y)=\left\{v \in L^{2}\left(\Omega ; H_{\#}^{1}(Y)\right): \exists \nabla_{y}^{B} v \in L^{2}(\Omega \times \mathcal{G})\right. \\
\text { and } \left.\int_{Y} v(x, y) \mathrm{d} y=0 \text { for a.e. } x \in \Omega\right\},
\end{array}
$$

where the supscript \# denotes $Y$-periodicity.

Definition 3.2. We say that a pair $\left(u, u^{1}\right) \in L^{2}\left(0, T ; H_{0}^{1}(\Omega)\right) \times L^{2}(0, T$; 
$\left.H_{0, \#}^{1, B}(\Omega \times Y)\right)$ is a weak solution of the problem (3.1)-(3.7) if

$$
\begin{aligned}
&-\int_{0}^{T} \int_{\Omega} u \frac{\partial \varphi}{\partial \tau} \mathrm{d} x \mathrm{~d} \tau+\int_{0}^{T} \int_{\Omega} A \nabla u \cdot \nabla \varphi \mathrm{d} x \mathrm{~d} \tau+\iint_{0} \int_{\Omega Y} B\left(\nabla u+\nabla_{y} u^{1}\right) \cdot \nabla \varphi \mathrm{d} x \mathrm{~d} y \mathrm{~d} \tau \\
&+\iint_{0} \int_{\mathcal{G}} \ell\left(\nabla^{B} u+\nabla_{y}^{B} u^{1}\right) \cdot \nabla \varphi \mathrm{d} x \mathrm{~d} \sigma \mathrm{d} \tau-\iint_{0} \int_{\Omega Y} u^{1} \frac{\partial \phi}{\partial \tau} \psi \mathrm{d} x \mathrm{~d} y \mathrm{~d} \tau \\
&+\int_{0}^{T} \int_{\Omega} B\left(\nabla u+\nabla_{y} u^{1}\right) \cdot \nabla_{y} \psi \phi \mathrm{d} x \mathrm{~d} y \mathrm{~d} \tau \\
&+\int_{0}^{T} \int_{\Omega} \int_{\mathcal{G}} \ell\left(\nabla^{B} u+\nabla_{y}^{B} u^{1}\right) \cdot \nabla_{y}^{B} \psi \phi \mathrm{d} x \mathrm{~d} \sigma \mathrm{d} \tau \\
&=\int_{\Omega} \bar{u}_{0} \varphi(x, 0) \mathrm{d} x+\iint_{\Omega Y} \bar{u}_{0}^{1} \phi(x, 0) \psi(y) \mathrm{d} x \mathrm{~d} y \\
&+\int_{0}^{T} \int_{\Omega} f \varphi \mathrm{d} x \mathrm{~d} \tau+\int_{0}^{T} \int_{\Omega Y} g \phi \psi \mathrm{d} x \mathrm{~d} y \mathrm{~d} \tau+\iint_{0}^{T} \int_{\Omega \mathcal{G}} h \phi \psi \mathrm{d} x \mathrm{~d} \sigma \mathrm{d} \tau
\end{aligned}
$$

for every $\varphi, \phi \in \mathcal{C}^{\infty}\left([0, T] ; \mathcal{C}_{c}^{\infty}(\Omega)\right), \varphi(\cdot, T)=\phi(\cdot, T)=0$ in $\Omega$, and $\psi \in$ $\mathcal{C}_{\#}^{\infty}(Y)$.

Theorem 3.3. Assume that $f, g, h, \bar{u}_{0}, \bar{u}_{0}^{1}, A, B$, $\ell$ satisfy (3.8), (3.9), and (3.14) and that the compatibility condition (3.13) is fulfilled by the sources $g$ and $h$. Then problem (3.1)-(3.7) admits a unique pair of solutions $\left(u, u^{1}\right) \in$ $\left[L^{2}\left(0, T ; H_{0}^{1}(\Omega)\right) \cap \mathcal{C}^{0}\left([0, T] ; L^{2}(\Omega)\right)\right] \times\left[L^{2}\left(0, T ; H_{0, \#}^{1, B}(\Omega \times Y)\right) \cap \mathcal{C}^{0}([0, T] ;\right.$ $\left.\left.L^{2}(\Omega \times Y)\right)\right]$.

Proof. Let us set

$$
H=L^{2}(\Omega) \times L^{2}(\Omega \times Y), \quad V=H_{0}^{1}(\Omega) \times H_{0, \#}^{1, B}(\Omega \times Y) ;
$$

and define, for every $\widetilde{u}=(u, v), \widetilde{w}=(w, \mathrm{v}) \in H($ or $\widetilde{u}=(u, v), \widetilde{w}=(w, \mathrm{v})$ 
$\in V$, respectively)

$$
\begin{aligned}
&(\widetilde{u}, \widetilde{w})_{H}:=\int_{\Omega} u w \mathrm{~d} x+\iint_{\Omega Y} v \mathrm{v} \mathrm{d} x \mathrm{~d} y \\
&(\widetilde{u}, \widetilde{w})_{V}:=(\widetilde{u}, \widetilde{w})_{H}+\int_{\Omega} \nabla u \cdot \nabla w \mathrm{~d} x+\iint_{\Omega Y} \nabla_{y} v \cdot \nabla_{y} \mathrm{v} \mathrm{d} x \mathrm{~d} y \\
&+\iint_{\Omega \mathcal{G}}\left(\nabla_{x}^{B} u+\nabla_{y}^{B} v\right) \cdot\left(\nabla_{x}^{B} w+\nabla_{y}^{B} \mathrm{v}\right) \mathrm{d} x \mathrm{~d} \sigma .
\end{aligned}
$$

We claim that $V$ and $H$ are Hilbert space endowed with the previously defined scalar products. Indeed, the product (3.17) is the standard one in $L^{2}(\Omega) \times L^{2}(\Omega \times Y)$. On the other hand, it can be easily verified that also (3.18) is a scalar product with respect to which $V$ is a complete space. To this purpose, we notice that (3.18) contains the sum of the scalar product in $H_{0}^{1}(\Omega)$ (which is complete) and the standard scalar product in $L^{2}\left(\Omega ; H_{\#}^{1}(Y)\right)$ (which is complete, too). Hence we have that, if $\left(u_{k}, u_{k}^{1}\right)_{k}$ is a Cauchy sequence in $V$, there exists $\left(u, u^{1}\right) \in H_{0}^{1}(\Omega) \times L^{2}\left(\Omega ; H_{\#}^{1}(Y)\right)$ such that $\left(u_{k}, u_{k}^{1}\right) \rightarrow\left(u, u^{1}\right)$ strongly in $H_{0}^{1}(\Omega) \times L^{2}\left(\Omega ; H_{\#}^{1}(Y)\right)$ and $u^{1}$ has null mean average on $Y$. Moreover, if $\left(u_{k}, u_{k}^{1}\right)_{k}$ is a Cauchy sequence in $V$ we have also that $\left(\nabla_{x}^{B} u_{k}+\nabla_{y}^{B} u_{k}^{1}\right)_{k}$ is a Cauchy sequence in $L^{2}(\Omega \times \mathcal{G})$. Therefore, it remains to verify that $\nabla_{x}^{B} u+\nabla_{y}^{B} u^{1}$ does exist and $\nabla_{x}^{B} u_{k}+$ $\nabla_{y}^{B} u_{k}^{1} \rightarrow \nabla_{x}^{B} u+\nabla_{y}^{B} u^{1}$, strongly in $L^{2}(\Omega \times \mathcal{G})$. However, by the completeness of $L^{2}(\Omega \times \mathcal{G})$, it follows that there exists $\vec{q} \in L^{2}(\Omega \times \mathcal{G})$ such that $\nabla_{x}^{B} u_{k}+\nabla_{y}^{B} u_{k}^{1} \rightarrow \vec{q}$ strongly in $L^{2}(\Omega \times \mathcal{G})$ and

$$
\begin{aligned}
& -\int_{\Omega} \int_{\mathcal{G}}\left(\nabla_{x}^{B} u+\nabla_{y}^{B} u^{1}\right) \cdot \Psi \mathrm{d} x \mathrm{~d} \sigma \mathrm{d} \tau= \\
& \iint_{\Omega}\left(u \operatorname{div}_{x}^{B} \boldsymbol{\Psi}+u^{1} \operatorname{div}_{y}^{B} \boldsymbol{\Psi}\right) \mathrm{d} x \mathrm{~d} \sigma \mathrm{d} \tau \longleftarrow \iint_{\Omega}\left(u_{k} \operatorname{div}_{x}^{B} \boldsymbol{\Psi}+u_{k}^{1} \operatorname{div}_{y}^{B} \boldsymbol{\Psi}\right) \mathrm{d} x \mathrm{~d} \sigma \\
& =-\int_{\Omega} \int_{\mathcal{G}}\left(\nabla_{x}^{B} u_{k}+\nabla_{y}^{B} u_{k}^{1}\right) \cdot \boldsymbol{\Psi} \mathrm{d} x \mathrm{~d} \sigma \longrightarrow-\iint_{\Omega \mathcal{G}} \vec{q} \cdot \boldsymbol{\Psi} \mathrm{d} x \mathrm{~d} \sigma,
\end{aligned}
$$

where $\boldsymbol{\Psi} \in \mathcal{C}_{0}^{\infty}\left(\Omega_{T} ; \mathcal{C}_{\#}^{\infty}(Y)\right)$ and the first equality follows by easy computations from formula (2.2). Hence, $\vec{q}=\nabla_{x}^{B} u+\nabla_{y}^{B} u^{1}$ and the claim is proved.

Finally, we also have that $V \subset H$ with continuous and dense injection (note that $\left.\mathcal{C}_{0}^{\infty}(\Omega \times Y) \subset V\right)$. 
Let us now define the bilinear and symmetric form $a: V \times V \rightarrow \mathbb{R}$ as

$$
\begin{aligned}
a(\widetilde{u}, \widetilde{w})=\int_{\Omega} A \nabla u \cdot \nabla w+ & \iint_{\Omega Y} B\left(\nabla u+\nabla_{y} v\right) \cdot\left(\nabla w+\nabla_{y} \mathrm{v}\right) \mathrm{d} x \mathrm{~d} y \\
& +\iint_{\Omega \mathcal{G}} \ell\left(\nabla^{B} u+\nabla_{y}^{B} v\right) \cdot\left(\nabla^{B} w+\nabla_{y}^{B} \mathrm{v}\right) \mathrm{d} x \mathrm{~d} y
\end{aligned}
$$

which satisfies

$$
\begin{gathered}
|a(\widetilde{u}, \widetilde{w})| \leq C\left(\|\nabla u\|_{L^{2}(\Omega)}\|\nabla w\|_{L^{2}(\Omega)}+\left\|\nabla_{y} v\right\|_{L^{2}(\Omega \times Y)}\left\|\nabla_{y} \mathrm{v}\right\|_{L^{2}(\Omega \times Y)}\right. \\
\left.+\left\|\nabla_{x}^{B} u+\nabla_{y}^{B} v\right\|_{L^{2}(\Omega \times \mathcal{G})}\left\|\nabla_{x}^{B} w+\nabla_{y}^{B} \mathrm{v}\right\|_{L^{2}(\Omega \times \mathcal{G})}\right) \leq C\|\widetilde{u}\|_{V}\|\widetilde{w}\|_{V} \\
a(\widetilde{u}, \widetilde{u}) \geq c\left(\|\nabla u\|_{L^{2}(\Omega)}^{2}+\left\|\nabla_{y} v\right\|_{L^{2}(\Omega \times Y)}^{2}+\left\|\nabla_{x}^{B} u+\nabla_{y}^{B} v\right\|_{L^{2}(\Omega \times \mathcal{G})}^{2}\right) \geq c\|\widetilde{u}\|_{V}^{2},
\end{gathered}
$$

where $c, C$ depend on $\gamma_{A}, \gamma_{B}, \gamma_{\ell}$ and the Poincaré constant and we have taken into account that, due to the $Y$-periodicity of $v$, we have

$$
\begin{array}{r}
\iint_{\Omega Y}\left|\nabla u+\nabla_{y} v\right|^{2} \mathrm{~d} x \mathrm{~d} y=\iint_{\Omega Y}\left(|\nabla u|^{2}+\left|\nabla_{y} v\right|^{2}+2 \nabla u \cdot \nabla_{y} v\right) \mathrm{d} x \mathrm{~d} y \\
=\iint_{\Omega Y}\left(|\nabla u|^{2}+\left|\nabla_{y} v\right|^{2}\right) \mathrm{d} x \mathrm{~d} y+2 \int_{\Omega} \nabla u \cdot\left(\int_{Y} \nabla_{y} v \mathrm{~d} y\right) \mathrm{d} x \\
=\iint_{\Omega Y}\left(|\nabla u|^{2}+\left|\nabla_{y} v\right|^{2}\right) \mathrm{d} x \mathrm{~d} y .
\end{array}
$$

Hence, $a$ is a continuous and coercive bilinear form on $V \times V$. Finally, let us denote by $V^{*}$ the topological dual space of $V$ and observe that $V \subset H \subset V^{*}$ is a so-called evolution triple (see [15, Def. 23.11]). Moreover, consider the linear and continuous operator $F \in L^{2}\left(0, T ; V^{*}\right)$ defined by

$$
\begin{aligned}
\langle F(t), \widetilde{\phi}\rangle=\int_{\Omega} f(x, t) \phi(x, t) \mathrm{d} x+ & =\iint_{\Omega Y} g(x, y, t) \Phi(x, y, t) \mathrm{d} x \mathrm{~d} y \\
& \quad+\iint_{\Omega \mathcal{G}} h(x, y, t) \Phi(x, y, t) \mathrm{d} x \mathrm{~d} \sigma,
\end{aligned}
$$

for a.e. $t \in(0, T)$ and every $\widetilde{\phi}=(\phi, \Phi) \in V$, where we denote by $\langle\cdot, \cdot\rangle$ the duality pairing between $V^{*}$ and $V$. 
Now let us consider the following abstract problem

$$
\begin{aligned}
& \text { find } \widetilde{u} \in L^{2}(0, T ; V) \cap \mathcal{C}^{0}([0, T] ; H) \text { such that } \widetilde{u}(0)=\left(\bar{u}_{0}, \bar{u}_{0}^{1}\right) \in H \\
& \text { and } \frac{d}{d t}(\widetilde{u}(t), \widetilde{\phi})_{H}+a(\widetilde{u}(t), \widetilde{\phi})=\langle F(t), \widetilde{\phi}\rangle \quad \forall \widetilde{\phi} \in V
\end{aligned}
$$

in the sense of distribution in $(0, T)$. We remark that (3.22) correspond to the abstract formulation of the problem (3.1)-(3.7); indeed, taking into account the compatibility condition (3.13), it is possible to prove that the weak form (3.16) can be equivalently rewritten using test function $\psi \in$ $\mathcal{C}_{\#}^{\infty}(Y)$ with null mean average on $Y$ (as it is usual in the periodic setting). Therefore, since by [15, Theorem 23A (a)] problem (3.22) admits a unique solution, the thesis is accomplished.

Remark 3.4. Previous theorem can be generalized to the case where the source terms are more general; i.e., we can assume that $F \in L^{2}\left(0, T ; V^{*}\right)$.

Remark 3.5. Notice that Theorem 3.3 can be generalized to the case in which the coefficients $\gamma \in L^{\infty}(\Omega)$ and $\alpha \in L^{\infty}(\mathcal{G})$ (with $\gamma(x) \geq \gamma_{0}$ a.e. in $\Omega$ and $\alpha(x) \geq \alpha_{0}$ a.e. on $\mathcal{G}$, for proper constants $\gamma_{0}, \alpha_{0}>0$ ) appear in front of the time derivatives in (3.1) and (3.2).

\section{Well-posedness for the parabolic-elliptic two- scale system}

This section is devoted to prove that, as a consequence of Theorem 3.3, we obtain the well-posedness of the following two-scale parabolic-elliptic system

$$
\begin{aligned}
& u_{t}-\operatorname{div}\left(A \nabla u+\int_{Y} B\left(\nabla_{y} u^{1}+\nabla_{x} u\right) \mathrm{d} y+\int_{\mathcal{G}} \ell\left(\nabla_{y}^{B} u^{1}+\nabla_{x}^{B} u\right) \mathrm{d} \sigma\right)=f, \text { in } \Omega_{T} ; \\
& -\operatorname{div}_{y}\left(B\left(\nabla_{y} u^{1}+\nabla_{x} u\right)\right)=g, \quad \text { in } \Omega_{T} \times\left(E_{\text {int }} \cup E_{\text {out }}\right) \text {; } \\
& \operatorname{div}_{y}^{B}\left(\ell\left(\nabla_{y}^{B} u^{1}+\nabla_{x}^{B} u\right)\right)=-\left[B\left(\nabla_{y} u^{1}+\nabla_{x} u\right) \cdot \nu\right]-h, \quad \text { in } \Omega_{T} \times \mathcal{G} ; \\
& u^{1}(x, \cdot, t) \\
& \text { is } Y \text {-periodic and with null mean average; } \\
& u=0 \text {, } \\
& \text { on } \partial \Omega \times(0, T) \text {; } \\
& u(x, 0)=\bar{u}_{0}(x),
\end{aligned}
$$


Definition 4.1. We say that a pair $\left(u, u^{1}\right) \in L^{2}\left(0, T ; H_{0}^{1}(\Omega)\right) \times L^{2}(0, T$; $\left.H_{0, \#}^{1, B}(\Omega \times Y)\right)$ is a weak solution of the problem (4.1)-(4.6) if

$$
\begin{aligned}
& -\int_{0}^{T} \int_{\Omega} u \frac{\partial \varphi}{\partial \tau} \mathrm{d} x \mathrm{~d} \tau+\int_{0}^{T} \int_{\Omega} A \nabla u \cdot \nabla \varphi \mathrm{d} x \mathrm{~d} \tau+\iint_{0} \int_{\Omega} B\left(\nabla u+\nabla_{y} u^{1}\right) \cdot \nabla \varphi \mathrm{d} x \mathrm{~d} y \mathrm{~d} \tau \\
& +\int_{0}^{T} \int_{\Omega} \int_{\mathcal{G}} \ell\left(\nabla^{B} u+\nabla_{y}^{B} u^{1}\right) \cdot \nabla \varphi \mathrm{d} x \mathrm{~d} \sigma \mathrm{d} \tau \\
& +\int_{0} \int_{\Omega Y} B\left(\nabla u+\nabla_{y} u^{1}\right) \cdot \nabla_{y} \psi \phi \mathrm{d} x \mathrm{~d} y \mathrm{~d} \tau+\iint_{0}^{T} \int_{\mathcal{G}} \ell\left(\nabla^{B} u+\nabla_{y}^{B} u^{1}\right) \cdot \nabla_{y}^{B} \psi \phi \mathrm{d} x \mathrm{~d} \sigma \mathrm{d} \tau \\
& =\int_{\Omega} u_{0} \varphi(x, 0) \mathrm{d} x+\int_{0}^{T} \int_{\Omega} f \varphi \mathrm{d} x \mathrm{~d} \tau+\iint_{0}^{T} \int_{\Omega Y} g \phi \psi \mathrm{d} x \mathrm{~d} y \mathrm{~d} \tau+\iint_{0}^{T} \int_{\Omega} h \phi \psi \mathrm{d} x \mathrm{~d} \sigma \mathrm{d} \tau,
\end{aligned}
$$

for every $\varphi, \phi \in \mathcal{C}^{\infty}\left([0, T] ; \mathcal{C}_{c}^{\infty}(\Omega)\right), \varphi(\cdot, T)=\phi(\cdot, T)=0$ in $\Omega$, and $\psi \in$ $\mathcal{C}_{\#}^{\infty}(Y)$.

Note also that, integrating (4.2) on $Y=E_{\text {int }} \cup E_{\text {out }}$, applying Gauss-Green formulas and taking into account (4.3), we obtain that, for a.e. $(x, t) \in \Omega_{T}$,

$$
\begin{aligned}
\int_{Y} g \mathrm{~d} y= & -\int_{Y} \operatorname{div}_{y}\left(B\left(\nabla_{y} u^{1}+\nabla_{x} u\right)\right) \mathrm{d} y=\int_{\mathcal{G}}\left[B\left(\nabla_{y} u^{1}+\nabla_{x} u\right) \cdot \nu\right] \mathrm{d} \sigma \\
& =-\int_{\mathcal{G}} \operatorname{div}_{y}^{B}\left(\ell\left(\nabla_{y}^{B} u^{1}+\nabla_{x}^{B} u\right)\right) \mathrm{d} \sigma-\int_{\mathcal{G}} h \mathrm{~d} \sigma=-\int_{\mathcal{G}} h \mathrm{~d} \sigma .
\end{aligned}
$$

Thus (4.8) is a compatibility condition for $g$ and $h$ (analogous to (3.13)), which now is an essential a-priori condition in order to have the wellposedness of problem (4.1)-(4.6).

Theorem 4.2. Assume that $f, g, h, \bar{u}_{0}, A, B, \ell$ satisfy (3.8) and (3.9) and that the compatibility condition (4.8) hold. Then the problem (4.1)-(4.6) admits a unique pair of solutions $\left(u, u^{1}\right) \in L^{2}\left(0, T ; H_{0}^{1}(\Omega)\right) \times L^{2}(0, T$; $\left.H_{0, \#}^{1, B}(Y)\right)$.

Proof. We will prove the existence of a pair of solution to problem (4.1)(4.6) via a relaxation technique. To this purpose let us consider, for every 
$\varepsilon>0$, the following problem

$$
\begin{aligned}
& u_{t}^{\varepsilon}-\operatorname{div}\left(A \nabla u^{\varepsilon}+\int_{Y} B\left(\nabla_{y} u^{\varepsilon, 1}+\nabla_{x} u^{\varepsilon}\right) \mathrm{d} y\right) \\
& -\operatorname{div}\left(\int_{\mathcal{G}} \ell\left(\nabla_{y}^{B} u^{\varepsilon, 1}+\nabla_{x}^{B} u^{\varepsilon}\right) \mathrm{d} \sigma\right)=f, \quad \text { in } \Omega_{T} ; \\
& \varepsilon u_{t}^{\varepsilon, 1}-\operatorname{div}_{y}\left(B\left(\nabla_{y} u^{\varepsilon, 1}+\nabla_{x} u^{\varepsilon}\right)\right)=g, \quad \text { in } \Omega_{T} \times\left(E_{\text {int }} \cup E_{\text {out }}\right) \text {; } \\
& \operatorname{div}_{y}^{B}\left(\ell\left(\nabla_{y}^{B} u^{\varepsilon, 1}+\nabla_{x}^{B} u^{\varepsilon}\right)\right)=-\left[B\left(\nabla_{y} u^{1}+\nabla_{x} u\right) \cdot \nu\right]-h \text {, in } \Omega_{T} \times \mathcal{G} ; \\
& u^{\varepsilon, 1}(x, \cdot, t) \\
& \text { is } Y \text {-periodic for a.e. }(x, t) \in \Omega_{T} \text {; } \\
& u^{\varepsilon}=0 \text {, } \\
& \text { on } \partial \Omega \times(0, T) \text {; } \\
& u^{\varepsilon, 1}(x, y, 0)=0 \text {, } \\
& \text { in } \Omega \times Y \text {; } \\
& u^{\varepsilon}(x, 0)=\bar{u}_{0}(x),
\end{aligned}
$$

Clearly, problem (4.9)-(4.15) is well-posed since, for $\varepsilon$ fixed, it can be treated as the problem (3.1)-(3.7), up to properly redefining the scalar products in (3.17) and (3.18) (due to the relaxation parameter $\varepsilon$, which appears explicitly in front of the time derivative in (4.10)). We recall also that, as a consequence of Remark 3.1, taking into account that (4.8) is fulfilled, it follows that $u^{\varepsilon, 1}$ has null mean average on $Y$, a.e. in $\Omega_{T}$. Moreover, by (3.16), we get the following energy estimate

$$
\begin{aligned}
& \frac{1}{2} \int_{0}^{T} \int_{\Omega} \frac{\partial\left(u^{\varepsilon}\right)^{2}}{\partial \tau} \mathrm{d} x \mathrm{~d} \tau+\int_{0} \int_{\Omega} A \nabla u^{\varepsilon} \cdot \nabla u^{\varepsilon} \mathrm{d} x \mathrm{~d} \tau \\
& \quad+\iint_{0}^{T} \int_{Y} B\left(\nabla u^{\varepsilon}+\nabla_{y} u^{\varepsilon, 1}\right) \cdot \nabla u^{\varepsilon} \mathrm{d} x \mathrm{~d} y \mathrm{~d} \tau \\
& +\iint_{0}^{T} \int_{\mathcal{G}} \ell\left(\nabla^{B} u^{\varepsilon}+\nabla_{y}^{B} u^{\varepsilon, 1}\right) \cdot \nabla u^{\varepsilon} \mathrm{d} x \mathrm{~d} \sigma \mathrm{d} \tau \\
& +\frac{\varepsilon}{2} \int_{0} \int_{\Omega Y}^{T} \frac{\partial\left(u^{\varepsilon, 1}\right)^{2}}{\partial \tau} \mathrm{d} x \mathrm{~d} y \mathrm{~d} \tau+\int_{0}^{T} \int_{\Omega Y} B\left(\nabla u^{\varepsilon}+\nabla_{y} u^{\varepsilon, 1}\right) \cdot \nabla_{y} u^{\varepsilon, 1} \mathrm{~d} x \mathrm{~d} y \mathrm{~d} \tau \\
& \quad+\iint_{0}^{T} \int_{\Omega \mathcal{G}} \ell\left(\nabla^{B} u^{\varepsilon}+\nabla_{y}^{B} u^{\varepsilon, 1}\right) \cdot \nabla_{y}^{B} u^{\varepsilon, 1} \mathrm{~d} x \mathrm{~d} \sigma \mathrm{d} \tau
\end{aligned}
$$




$$
=\int_{0}^{T} \int_{\Omega} f u^{\varepsilon} \mathrm{d} x \mathrm{~d} \tau+\iint_{0}^{T} \int_{\Omega} g u^{\varepsilon, 1} \mathrm{~d} x \mathrm{~d} y \mathrm{~d} \tau+\iint_{0}^{T} \int_{\mathcal{G}} h u^{\varepsilon, 1} \mathrm{~d} x \mathrm{~d} \sigma \mathrm{d} \tau .
$$

Taking into account (3.8) and the fact that $\left(\nabla^{B} u^{\varepsilon}+\nabla_{y}^{B} u^{\varepsilon, 1}\right) \cdot \nabla u^{\varepsilon}=$ $\left(\nabla^{B} u^{\varepsilon}+\nabla_{y}^{B} u^{\varepsilon, 1}\right) \cdot \nabla^{B} u^{\varepsilon}$, by Young inequality it follows

$$
\begin{array}{r}
\sup _{t} \int_{\Omega}\left(u^{\varepsilon}\right)^{2} \mathrm{~d} x+\varepsilon \sup _{t} \int_{\Omega} \int_{Y}\left(u^{\varepsilon, 1}\right)^{2} \mathrm{~d} x \mathrm{~d} y+\int_{0} \int_{\Omega}\left|\nabla u^{\varepsilon}\right|^{2} \mathrm{~d} x \mathrm{~d} \tau \\
+\iint_{\Omega} \int_{Y}\left|\nabla_{y} u^{\varepsilon, 1}\right|^{2} \mathrm{~d} x \mathrm{~d} y \mathrm{~d} \tau+\iint_{0} \int_{\mathcal{G}}\left|\nabla^{B} u^{\varepsilon}+\nabla_{y}^{B} u^{\varepsilon, 1}\right|^{2} \mathrm{~d} x \mathrm{~d} \sigma \mathrm{d} \tau \\
\leq C\left(\left\|u_{0}\right\|_{L^{2}(\Omega)}^{2}+\|f\|_{L^{2}\left(\Omega_{T}\right)}^{2}+\|g\|_{L^{2}\left(\Omega_{T} \times Y\right)}^{2}+\|h\|_{L^{2}\left(\Omega_{T} \times \mathcal{G}\right)}^{2}\right) \\
+C \delta\left(\left\|\nabla u^{\varepsilon}\right\|_{L^{2}\left(\Omega_{T}\right)}^{2}+\left\|\nabla_{y} u^{\varepsilon, 1}\right\|_{L^{2}\left(\Omega_{T} \times Y\right)}^{2}\right)
\end{array}
$$

where $C$ depends on $\mathcal{G}, \gamma_{A}, \gamma_{B}, \gamma_{C}$, the Poincaré's and the trace inequality constants. We remark also that, in the left-hand side we use the following inequality

$$
\iint_{0}^{T} \int_{\Omega Y}\left|\nabla u^{\varepsilon}+\nabla_{y} u^{\varepsilon, 1}\right|^{2} \mathrm{~d} x \mathrm{~d} y \mathrm{~d} \tau=\iint_{0}^{T} \int_{\Omega}\left(\left|\nabla u^{\varepsilon}\right|^{2}+\left|\nabla_{y} u^{\varepsilon, 1}\right|^{2}\right) \mathrm{d} x \mathrm{~d} y \mathrm{~d} \tau,
$$

which is a consequence of the $Y$-periodicity of $u^{\varepsilon, 1}$ and can be proved as in (3.21). This implies that the left-hand side of (4.17) is uniformly bounded with respect to $\varepsilon$; i.e.,

$$
\begin{aligned}
& \sup _{t} \int_{\Omega}\left(u^{\varepsilon}\right)^{2} \mathrm{~d} x+\varepsilon \sup _{t} \int_{\Omega} \int_{Y}\left(u^{\varepsilon, 1}\right)^{2} \mathrm{~d} x \mathrm{~d} y+\int_{0}^{T} \int_{\Omega}\left|\nabla u^{\varepsilon}\right|^{2} \mathrm{~d} x \mathrm{~d} \tau \\
& \quad+\int_{0} \int_{\Omega} \int_{Y}\left|\nabla_{y} u^{\varepsilon, 1}\right|^{2} \mathrm{~d} x \mathrm{~d} y \mathrm{~d} \tau+\iint_{0}^{T} \int_{\Omega}\left|\nabla^{B} u^{\varepsilon}+\nabla_{y}^{B} u^{\varepsilon, 1}\right|^{2} \mathrm{~d} x \mathrm{~d} \sigma \mathrm{d} \tau \leq C,
\end{aligned}
$$

where $C$ is a strictly positive constant independent of $\varepsilon$. As a consequence of this estimate, we obtain that there exist $u \in L^{2}\left(0, T ; H_{0}^{1}(\Omega)\right), u^{1} \in$ 
$L^{2}\left(\Omega_{T} ; H_{\#}^{1}(Y)\right)$ with null mean average on $Y$ and $\vec{q} \in L^{2}\left(\Omega_{T} \times \mathcal{G}\right)$ such that, up to a subsequence,

$$
\begin{array}{lr}
u^{\varepsilon} \rightarrow u & w-L^{2}\left(\Omega_{T}\right) ; \\
u^{\varepsilon, 1} \rightarrow u^{1} & w-L^{2}\left(\Omega_{T} \times Y\right) ; \\
\nabla_{x} u^{\varepsilon} \rightarrow \nabla_{x} u & w-L^{2}\left(\Omega_{T}\right) ; \\
\nabla_{y} u^{\varepsilon, 1} \rightarrow \nabla_{y} u^{1} & w-L^{2}\left(\Omega_{T} \times Y\right) ; \\
\nabla_{x}^{B} u^{\varepsilon}+\nabla_{y}^{B} u^{\varepsilon, 1} \rightarrow \vec{q} & w-L^{2}\left(\Omega_{T} \times \mathcal{G}\right) .
\end{array}
$$

Therefore, we can pass to the limit, for $\varepsilon \rightarrow 0$, in the weak formulation of $(4.9)-(4.15)$, which is analogous to (3.16) written for $\left(u^{\varepsilon}, u^{1, \varepsilon}\right)$, where $\int_{0}^{T} \int_{\Omega} \int_{Y} u^{1} \frac{\partial \phi}{\partial \tau} \psi \mathrm{d} x \mathrm{~d} y \mathrm{~d} \tau$ is replaced by $\varepsilon \int_{0}^{T} \int_{\Omega} \int_{Y} u^{\varepsilon, 1} \frac{\partial \phi}{\partial \tau} \psi \mathrm{d} x \mathrm{~d} y \mathrm{~d} \tau \rightarrow 0$. Finally, following analogous computations as in (3.19), we obtain that $\vec{q}=\nabla_{x}^{B} u+\nabla_{y}^{B} u^{1}$. Thus, the existence of a weak solution for the problem (4.1)-(4.6) is proved.

In order to prove uniqueness, we have only to take into account the linearity of problem (4.1)-(4.6) and the associated energy estimate which, carrying on the proper integration, turns out to be exactly the same as (4.19) having set $\varepsilon=0$.

\section{References}

[1] G. Allaire, A. Damlamian, and U. Hornung. Two-scale convergence on periodic surfaces and applications. Proceedings of the International Conference on Mathematical Modelling of Flow through Porous Media, (1995) 15-25.

[2] M. Amar, D. Andreucci, and D. Bellaveglia. Homogenization of an alternating Robin-Neumann boundary condition via time-periodic unfolding. Nonlinear Anal. - Theor., 153 (2017) 56-77.

[3] M. Amar, D. Andreucci, and D. Bellaveglia. The time-periodic unfolding operator and applications to parabolic homogenization. Atti Accad. Naz. Lincei Rend. Lincei Mat. Appl., 28 (2017) 663-700.

[4] M. Amar, D. Andreucci, P. Bisegna, and R. Gianni. A hierarchy of models for the electrical conduction in biological tissues via twoscale convergence: The nonlinear case. Differ. Integral Equ., (9-10) 26 (2013) 885-912. 
[5] M. Amar, D. Andreucci, R. Gianni, and C. Timofte. Concentration and homogenization in electrical conduction in heterogeneous media involving the Laplace-Beltrami operator. Submitted (2019).

[6] M. Amar, I. de Bonis, and G. Riey. Homogenization of elliptic problems involving interfaces and singular data. Submitted (2019).

[7] M. Amar and R. Gianni. Error estimate for a homogenization problem involving the Laplace-Beltrami operator. Math. Mech. Complex Syst., (1) 6 (2018) 41-59.

[8] M. Amar and R. Gianni. Laplace-Beltrami operator for the heat conduction in polymer coating of electronic devices. Discrete Contin. Dynam. Systems - Series B, (4) 23 (2018) 1739-1756.

[9] R. Bunoiu and C. Timofte. Homogenization of a thermal problem with flux jump. Netw. Heterog. Media, (4) 11 (2016) 545-562.

[10] D. Cioranescu, A. Damlamian, P. Donato, G. Griso, and R. Zaki. The periodic unfolding method in domains with holes. SIAM J. Math. Anal., (2) 44 (2012) 718-760.

[11] D. Cioranescu, A. Damlamian, and G. Griso. The periodic unfolding method in homogenization. SIAM J. Math. Anal., (4) 40 (2008) 15851620 .

[12] P. Donato and K. H. Le Nguyen. Homogenization of diffusion problems with a nonlinear interfacial resistance. NoDEA Nonlinear Differ. Equ. Appl., (5) 22 (2015) 1345âĂŞ1380.

[13] P. A. Raviart and J. M. Thomas. Introduction á l'analyse numérique des équations aux dérivées partielles. (1983) Masson, Paris, France.

[14] C. Timofte. Multiscale analysis of diffusion processes in composite media. Comput. Math. Appl., (9) 66 (2013) 1573-1580.

[15] E. Zeidler. Nonlinear functional analysis and its application. II/A (1990) Springer-Verlag, Berlin. 